\title{
Edoxaban improves venous thrombosis via increasing hydrogen sulfide and homocysteine in rat model
}

\author{
WEI SONG ${ }^{1,2}$, HONGBO CI ${ }^{2}$, GUANGLEI TIAN ${ }^{2}$, YANJUN ZHANG ${ }^{3}$ and XIAOHU $\mathrm{GE}^{2}$ \\ ${ }^{1}$ Postgraduate College of Xinjiang Medical University, Urumqi, Xinjiang 830054; Departments of ${ }^{2}$ Vascular Surgery \\ and ${ }^{3}$ Clinical Medical Research Center, People's Hospital of Xinjiang Uygur Autonomous Region, \\ Urumqi, Xinjiang 830011, P.R. China
}

Received August 31, 2016; Accepted May 25, 2017

DOI: $10.3892 / \mathrm{mmr} .2017 .7574$

\begin{abstract}
Anticoagulant therapy is prescribed to millions of patients worldwide for the prevention and treatment of venous thrombosis. Evidence has indicated that edoxaban is a potential drug of oral anticoagulant in the acute treatment of venous thromboembolism. Hydrogen sulfide and homocysteine plasma concentration are indicators of cardiovascular and neurovascular disease risk factors that have attracted considerable attention for regulation of vascular health and homeostasis. However, the molecular mechanism of edoxaban-mediated differences of hydrogen sulfide and homocysteine has not been investigated in the progression of venous thrombosis. In the present study, the authors analyzed the phosphoinositide 3-kinase (PI3K)/protein kinase B (AKT) signaling pathway in the vein endothelial cells and expression levels of hydrogen sulfide and homocysteine. Homocysteine-hydrogen sulfide metabolism through transsulfuration and that transsulfuration capacity and hydrogen sulfide availability have been investigated both in vitro and in vivo following treatment with edoxaban. Matrix metalloproteinase (MMP) activation and cystathionine $\beta$-synthase (CBS) and cystathionine $\gamma$-lyase (CGL) levels were studied in a cell model and rat model of vein thrombosis prior and post treatment of edoxaban. The therapeutic effects of edoxaban for rats with vein thrombosis were determined by clinical diagnose scores. The results demonstrated that edoxaban increased expression levels of hydrogen sulfide and homocysteine in microvascular endothelial cells. It was observed that the transsulfuration enzymes, CBS and CGL levels were upregulated in murine microvascular endothelial cells. The MMP-9 expression level and activity and homocysteine-hydrogen sulfide metabolism were increased in murine microvascular endothelial cells following
\end{abstract}

Correspondence to: Professor Xiaohu Ge, Department of Vascular Surgery, People's Hospital of Xinjiang Uygur Autonomous Region, 91 Tianchi Road, Urumqi, Xinjiang 830011, P.R. China

E-mail: gexiaohuxj@163.com

Key words: edoxaban, venous thrombosis, hydrogen sulfide, homocysteine, phosphoinositide 3-kinase/protein kinase B edoxaban treatment. In addition, CBS and CGL activities were upregulated in murine microvascular endothelial cells and a rat model of venous thrombosis following treatment with edoxaban. Furthermore, it was observed that edoxaban increased PI3K and AKT expression both in vitro and in vivo. In addition, edoxaban significantly improved endothelial injury and inhibited thrombosis factors expression in rat model of venous thrombosis. In conclusion, these findings suggested that edoxaban can improve venous thrombosis by decreasing hydrogen sulfide and homocysteine through the PI3K/AKT signaling pathway.

\section{Introduction}

Venous thromboembolism is a severe life-threatening disease that comprises deep vein thrombosis and pulmonary embolism that significantly affects the life quality of patients with venous thrombosis $(1,2)$. Venous thrombosis is the most common disorder disease, which associates with various molecular mechanisms and diverse processes (3). A previous study indicated that anticoagulation treatments are the most common used and efficient drugs for the prevention and treatment adults and children undergone catheter-related thrombosis in clinical (4). Many factors can induce the pathology of venous thromboembolism main including vascular endothelial cell injury, blood flow state changes and the increase of blood clotting, which can easily cause venous thrombosis (5). Importantly, venous thromboembolism can lead to a large number diseases, such as pulmonary embolism, neurologic diseases and skin disease (6-8). Previously, changes in hydrogen sulfide and homocysteine expression levels lead to significant clinical directive significance for the patients with vein thrombosis $(9,10)$.

Currently, hydrogen sulfide can impact function of platelets that is enzymatically generated by platelets (11). Evidence has suggested that platelets serve important roles in hemostasis and thrombosis via circulating blood elements through activated by various stimuli (12). Hydrogen sulfide from slow-release compounds inhibits aggregation and exerts anti-thrombotic effects of platelets to in vivo (13). Theoretically, hydrogen sulfide acts as a potentially negative regulator of thrombosis through regulation of platelet activation (14). Research has also indicated that hydrogen sulfide is identified 
as an indicator of physiology of venous thromboembolism, myocardial infarction and stroke (15). In addition, the association of cystathionine $\gamma$-lyase (CGL) enzyme can be used to evaluate the risk of retinal vein thrombosis (16). Moreover, a previous study suggested that hydrogen sulfide generation in the endothelium may be a potential target for the treatment of arterial thrombosis (17). Therefore, the authors assumed that hydrogen sulfide generation may contribute to underlying the pathological characteristics of vein thrombosis.

The functions of hemostasis as an independent risk factor for venous thrombosis still remain controversial. Previous studies have presented the relationships between homocysteine and deep vein thrombosis $(18,19)$. The correlation of homocysteine and vascular thrombosis disease has been investigated in several clinical studies that demonstrated an increasing plasma total homocysteine $(20,21)$. Ravari et al (22) indicated that serum homocysteine in deep venous thrombosis is higher than in healthy volunteers. Potential mechanisms of homocysteine-mediated thrombosis and angiostasis in vascular pathobiology have been reviewed in a previous study (23). Therefore, a large number of drug targets for the regulation of homocysteine plasma concentration and its regulatory enzyme were put forward to improve the treatment of pathological characteristics of vein thrombosis.

In the present study, the authors investigated the efficacies and molecular mechanism of edoxaban-mediated differentiation changes of hydrogen sulfide and homocysteine in vein endothelial cells and in rats with venous thrombosis. The data suggested that edoxaban can significantly improve pathological characteristics of venous thrombosis by decreasing hydrogen sulfide and homocysteine through the phosphoinositide 3-kinase (PI3K)/protein kinase B (AKT) signaling pathway. These findings indicated that edoxaban may be a potential anti-thrombosis drug for the treatment of venous thrombosis.

\section{Materials and methods}

Ethics statement. The rat experiments were implemented legitimately according to the Guide for the Care and Use of Laboratory Animals, and were approved by the People's Hospital of Xinjiang Uygur Autonomous Region (Urumqi, China). All patients were recruited voluntarily and signed an agreement in a written form. All surgical operations and euthanasia were made to minimize suffering.

Cells culture and reagents. Vein endothelial cells (VECs) were isolated from Sprague-Dawley rats $(n=40$; male; age, 12 months; weight, 250-300 g) with ferric chloride-induced liver fibrosis. VECs were cultured in Dulbecco's modified Eagle's medium (Sigma-Aldrich; Merck KGaA, Darmstadt, Germany) supplemented with 10\% FBS (Invitrogen; Thermo Fisher Scientific, Inc., Waltham, MA, USA). All cells were cultured in a $37^{\circ} \mathrm{C}$ humidified atmosphere of $5 \% \mathrm{CO}_{2}$.

RNA isolation and reverse transcription-quantitative polymerase chain reaction ( $R T-q P C R)$. Total RNA from VECs was extracted using RNAeasy Mini kit (Qiagen $\mathrm{GmbH}$, Hilden, Germany). A total of $1 \mu \mathrm{g}$ total RNA was used to synthesize cDNA by performing the SuperScript II First-Strand Synthesis system (Invitrogen; Thermo Fisher Scientific, Inc.) according to the manufacturer's instructions. Gene expression levels were measured by RT-qPCR. All the forward and reverse primers were synthesized by Invitrogen; Thermo Fisher Scientific, Inc. All mRNA was quantified by using Power SYBR Green Master Mix (Applied Biosystems; Thermo Fisher Scientific, Inc.). Primers were designed as follows: Hydrogen sulfide, forward, 5'-CAAAGGTGGATCAGATTCAAG-3' and reverse, 5'-GGT GAGCATTATCACCCAGAA-3'; homocysteine, forward, 5'-CAAAGGTGGATCAGATTCAAG-3' and reverse, 5'-GGT GAGCATTATCACCCAGAA-3'; $\beta$-actin, forward, 5'-CAA AGGTGGATCAGATTCAAG-3' and reverse, 5'-GGTGAG CATTATCACCCAGAA-3'. The PCR thermocycling conditions were as follows: $95^{\circ} \mathrm{C}$ for $5 \mathrm{~min}$, then 35 cycles of $95^{\circ} \mathrm{C}$ for $20 \mathrm{sec}, 58^{\circ} \mathrm{C}$ for $20 \mathrm{sec}$ and $72^{\circ} \mathrm{C}$ for $20 \mathrm{sec}$, and a final extension at $72^{\circ} \mathrm{C}$ for $5 \mathrm{~min}$. $\beta$-actin was used as the internal reference gene. Relative mRNA expression levels were calculated by $2^{-\Delta \Delta \mathrm{Cq}}(24)$. The results analyzed in triplicate according to the $2^{-\Delta \Delta \mathrm{Cq}}$ method as the n-fold way compared to control.

Western blotting. Vein endothelial cells were isolated from experimental mice and homogenized in lysate buffer containing protease-inhibitor and were centrifuged at $7,104 \mathrm{x} \mathrm{g}$ at $4^{\circ} \mathrm{C}$ for $10 \mathrm{~min}$. The supernatant of mixture was used for analysis of purpose protein. Proteins were extracted using NP40 lysis buffer (Beyotime Institute of Biotechnology, Haimen, China) and quantified using the standard bicinchoninic acid method (Thermo Fisher Scientific, Inc.). SDS-PAGE assays were performed as previously described (25). For western blotting, primary antibodies against cystathionine $\beta$-synthase (CBS; 1:1,000; cat no. ab96252), CGL (1:1,000; cat no. ab131052), E26 transformation-specific (ETS; 1:1,000; cat no. ab26096), PI3K (1:1,000; cat no. ab74136), AKT (1:1,000; cat no. ab38449), phospho-AKT (1:1,000; cat no. ab8805), matrix metalloproteinase-9 (MMP-9; 1:5,000; cat no. ab38898) (all from Abcam, Cambridge, UK), ADP-dependent glucokinase precursor (1:1,000; cat no. ABP56684; Abbkine Scientific Co., Ltd., Wuhan, China), plasminogen activator inhibitors (PAIs; 1:1,000; cat no. ab169550), von Willebrand factor (vWf; 1:500; cat no. ab6994), thromboxane-A2 (TH-A2; 1:1,000; cat no. ab137607) and $\beta$-actin (1:1,000; cat no. ab8226) (all from Abcam) were added after blocking (5\% skimmed milk) for $1 \mathrm{~h}$ at $37^{\circ} \mathrm{C}$ and then incubated with horseradish peroxidase-conjugated rabbit anti-rat IgG secondary antibodies (1:1,000; cat no. ab6734; Abcam) for $24 \mathrm{~h}$ at $4^{\circ} \mathrm{C}$. The results were visualized using the ChemiDoc XRS system with Quantity One software v2.9 (Bio-Rad Laboratories, Inc., Hercules, CA, USA). Protein expression was analyzed using BandScan 5.0 software (Glyko, Inc., Novato, CA, USA) and all experiments were repeated at least three times.

Animals study. The Sprague-Dawley male rats ( $\mathrm{n}=40$; male; age, 12 months; weight, 250-300 g) were purchased from Vital River Laboratory Animal Technology Co., Ltd. (Shanghai, China). All mice were free to access food and water, and housed with a $12 \mathrm{~h}$ light-dark artificial cycle. To develop venous thrombosis, ferric chloride was used to induce Sprague-Dawley rat venous thrombosis according to a previous study (25). Rats with venous thrombosis were divided into two groups and received treatment with edoxaban $(10 \mathrm{mg} / \mathrm{kg})$ or the same dose of PBS. The treatments were continued for 60 days and 
received drugs once a day. On day 60 , rats were sacrificed and the venous samples were obtained for further analysis.

CBS and CGL activities. Activities of CBS and CGL were analyzed in microvascular endothelial cells following treatment with edoxaban $(10 \mathrm{mg} / \mathrm{l})$ according previous reports $(26,27)$.

Histological analysis. The cerebral vein was isolated from experimental rats and then fixed with $4 \%$ paraformaldehyde and permeabilized by incubating with absolute ethanol. Antigen retrieval was performed before performing immunofluorescent staining. The cerebral vein sections were incubated with goat anti-murine primary antibodies (Cell Signaling Technology, Inc., Danvers, MA, USA) for $12 \mathrm{~h}$ at $4^{\circ} \mathrm{C}$. Fluorescent labeled Alexa Fluor 488 (Molecular Probes; Thermo Fisher Scientific, Inc.) rabbit anti-goat secondary antibodies (1:1,000; cat no. ab6741; Abcam) were used to stain the proteins. The histological sections were subsequently scanned by a confocal microscope (Zeiss 510; Zeiss GmbH, Jena, Germany).

ELISA. The plasma concentration levels of hydrogen sulfide (cat no. 0-009160) and homocysteine (cat no. 0-011940) (Jianglai Biotechnology Co., Ltd., Shanghai, China) in patients with venous thrombosis and rat model of venous thrombosis were analyzed by commercialized ELISA kits. The operational procedures were performed by the manufacturer's instructions. The results were analyzed using the ELISA reader system (Bio-Rad Laboratories, Inc.).

Statistical analysis. All data are presented as mean \pm standard error of the mean and was conducted in triplicate. Statistical differences between experimental groups were analyzed by Student's t-test. $\mathrm{P}<0.05$ was considered to indicate a statistically significant difference.

\section{Results}

Expression levels of hydrogen sulfide and homocysteine in patients with venous thrombosis and rat model of venous thrombosis. To investigate the molecular mechanism of hydrogen sulfide and homocysteine in the progression of venous thrombosis, a murine model of venous thrombosis were established and patients with venous thrombosis were recruited to analyze the expression levels of hydrogen sulfide and homocysteine. Plasma concentration levels of hydrogen sulfide were downregulated in patients with venous thrombosis compared to healthy volunteers (Fig. 1A). Expression levels of homocysteine were higher in patients with venous thrombosis compared to healthy volunteers (Fig. 1B). Results demonstrated expression levels of hydrogen sulfide and homocysteine (Fig. 1C and D) in the rat model of venous thrombosis (MMVH) presented the same downregulation in expression as CBS and CGL (Fig. 1G). It was observed that CBS and CGL expression levels and activities were markedly downregulated in patients with venous thrombosis (Fig. 1E and F). Results also demonstrated that CBS and CGL expression levels and activities were also significantly downregulated in a rat model of venous thrombosis (Fig. $1 \mathrm{G}$ and $\mathrm{H}$ ). Taken together, these data suggested that hydrogen sulfide and homocysteine are downregulated in patients with venous thrombosis.

Edoxaban regulates hydrogen sulfide and homocysteine levels in vein endothelial cells. To explore the underlying molecular mechanism of upregulation of hydrogen sulfide and homocysteine, vein endothelial cells were used to analyze the efficacy of edoxaban in vitro. As presented in Fig. 2A and B, edoxaban treatment upregulated hydrogen sulfide and downregulated homocysteine mRNA expression levels may be regulated by methionine metabolism disorder in vein endothelial cells. In addition, transsulfuration enzymes, CBS and CGL levels were upregulated in edoxaban-treated murine microvascular endothelial cells (Fig. 2C-E). Results demonstrated that CBS and CGL activities were upregulated in rat microvascular endothelial cells, and following treatment with edoxaban (Fig. 2F and G). Furthermore, edoxaban enhanced the activity of vein endothelial cells following $48 \mathrm{~h}$ treatment (Fig. 2H). Taken together, these data suggested that edoxaban upregulates hydrogen sulfide and homocysteine expression levels in vein endothelial cells.

Edoxaban regulates the hydrogen sulfide and homocysteine activities through MMP9-induce PI3K/AKT signaling pathway. MMP-9 expression level and activity and homocysteine-hydrogen sulfide metabolism were increased in murine microvascular endothelial cells following edoxaban incubation. Expression levels of PI3K and AKT were increased in edoxaban-incubated microvascular endothelial cells (Fig. 3A and B). Phosphorylation of AKT also was upregulated in microvascular endothelial cells following edoxaban incubation (Fig. 3C). MMP-9 expression level was also increased in edoxaban-treated microvascular endothelial cells (Fig. 3D). In addition, the edoxaban induced increase of PI3K, AKT and MMP-9 levels was canceled by the PI3K inhibitor LY294002 (PI3KIR) in microvascular endothelial cells (Fig. 3E-G). Notably, PI3KIR markedly inhibited hydrogen sulfide and homocysteine activities in microvascular endothelial cells (Fig. 3H). Taken together, these findings indicated that edoxaban can regulate the hydrogen sulfide and homocysteine activities through the MMP9-induced PI3K/AKT signaling pathway.

The in vivo effects of edoxaban on rat with ferric chloride-induced venous thrombosis. In a rat glycyrrhizin-induced venous thrombosis model, the authors examined the therapeutic effects of edoxaban determined by changes of venous lesions. It was first demonstrated that edoxaban significantly improved ferric chloride-induced venous thrombosis in rat with venous thrombosis (Fig. 4A). Plasma concentration levels of hydrogen sulfide and homocysteine were also increased in edoxaban-treated rat with ferric chloride-induced venous thrombosis (Fig. 4B and C). Additionally, transsulfuration enzymes, CBS and CGL activities in microvascular endothelial cells were upregulated in experimental mice following edoxaban treatment (Fig. 4D-F). Furthermore, it was observed that ADP, PAIs, vWF and TH-A2 protein levels were markedly downregulated in microvascular endothelial cells following edoxaban treatment compared to the control (Fig. 4G). Importantly, apolipoprotein and thrombomodulin levels 
A

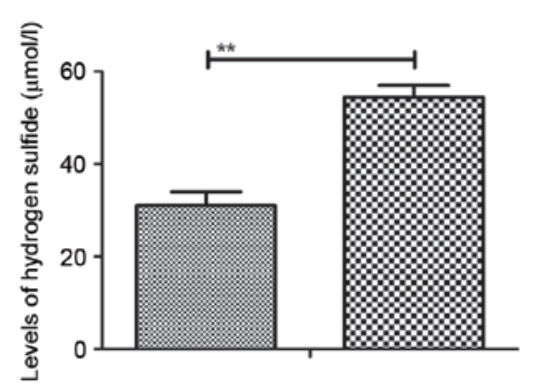

C

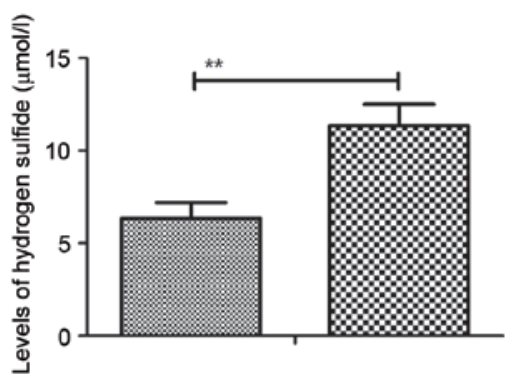

E

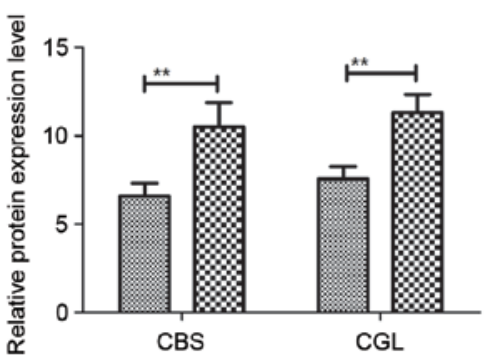

G

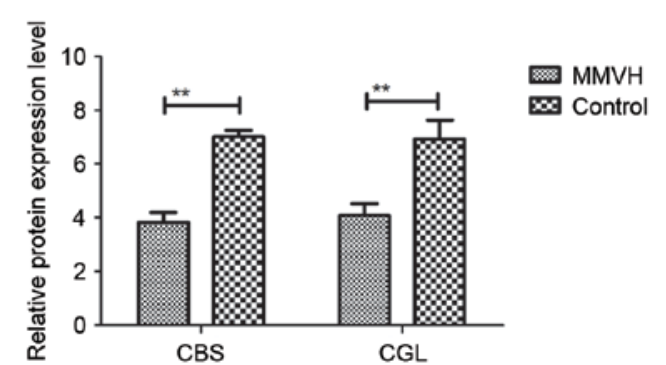

B

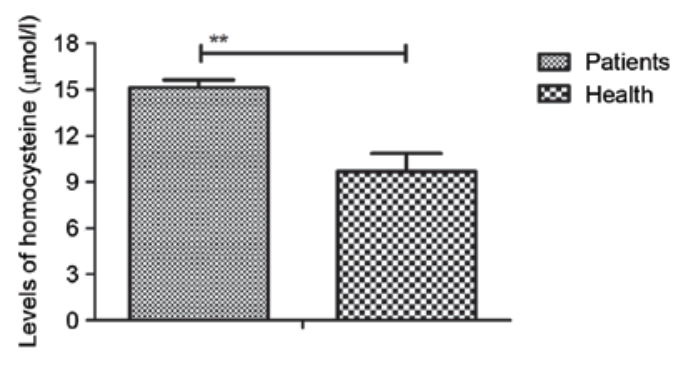

D

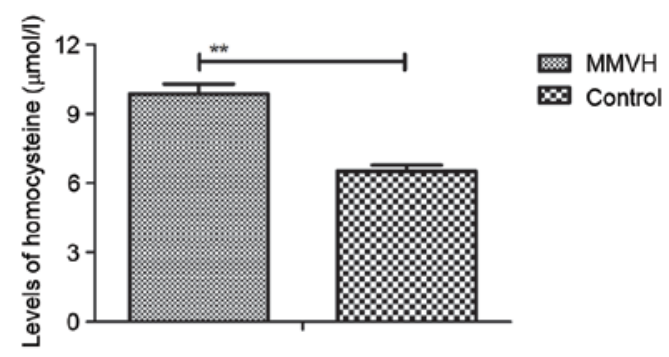

$\mathrm{F}$

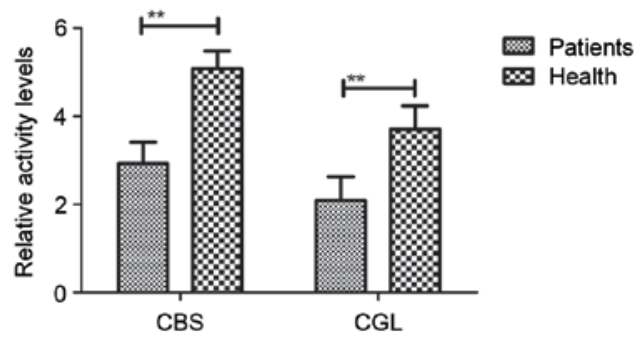

$\mathrm{H}$

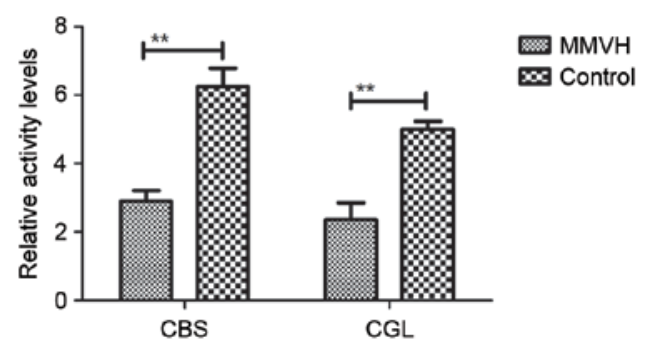

Figure 1. Analysis of hydrogen sulfide and homocysteine expression levels in the progression of venous thrombosis. Plasma concentration levels of (A) hydrogen sulfide and (B) homocysteine in patients with venous thrombosis. Plasma concentration levels of (C) hydrogen sulfide and (D) homocysteine in rat with venous thrombosis. (E) Relative CBS and CGL protein expression in in patients with venous thrombosis. (F) Relative CBS and CGL activity in patients with venous thrombosis. (G) Relative CBS and CGL protein expression in in rat with venous thrombosis. (H) Relative CBS and CGL activity in rat with venous thrombosis. The data are presented as the mean \pm standard error of mean of three independent experiments. ${ }^{* *} \mathrm{P}<0.01$ as indicated. CBS, cystathionine beta-synthase; CGL, cystathionine $\gamma$-lyase; MMVH, murine model of venous thrombosis.

were decreased in microvascular endothelial cells following edoxaban treatment compared to control (Fig. 4H). Taken together, these results demonstrated that edoxaban is an efficient anti-thrombosis drug for the treatment of ferric chloride-induced venous thrombosis.

\section{Discussion}

Currently, more and more new generations of target-specific oral anticoagulants are being developed to prevent and treat the increasing patients at risk of venous thrombosis $(28,29)$. These oral anticoagulants present different functions in the treatment of patients with atrial fibrillation (30). Like many new generations of target-specific anticoagulants, edoxaban is a fast-acting oral anticoagulant, which has been used to prevent stroke in patients with nonvalvular atrial fibrillation and in treating acute venous thrombosis through selectively inhibiting factor Xa (31). Edoxaban is currently approved in Japan and the USA for the prevention and treatment of venous thromboembolism following major orthopaedic 
A

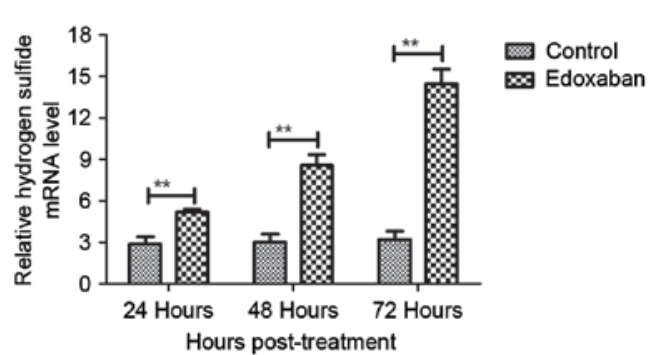

C

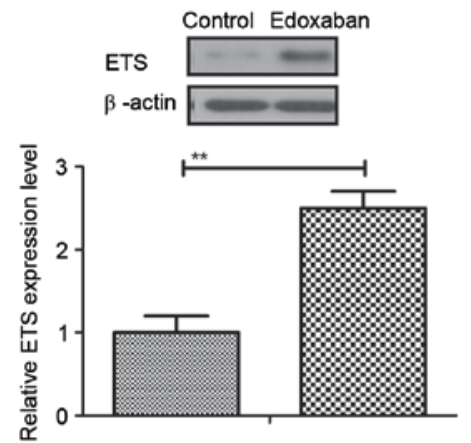

E
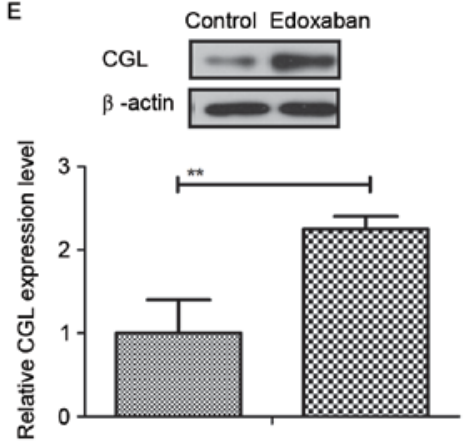

G
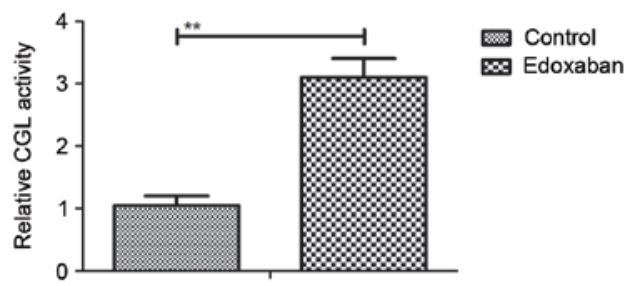

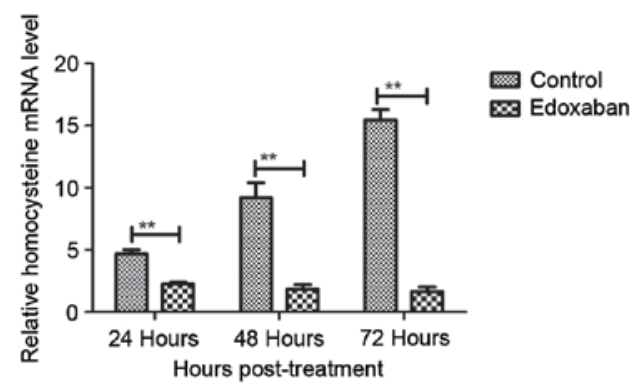

D
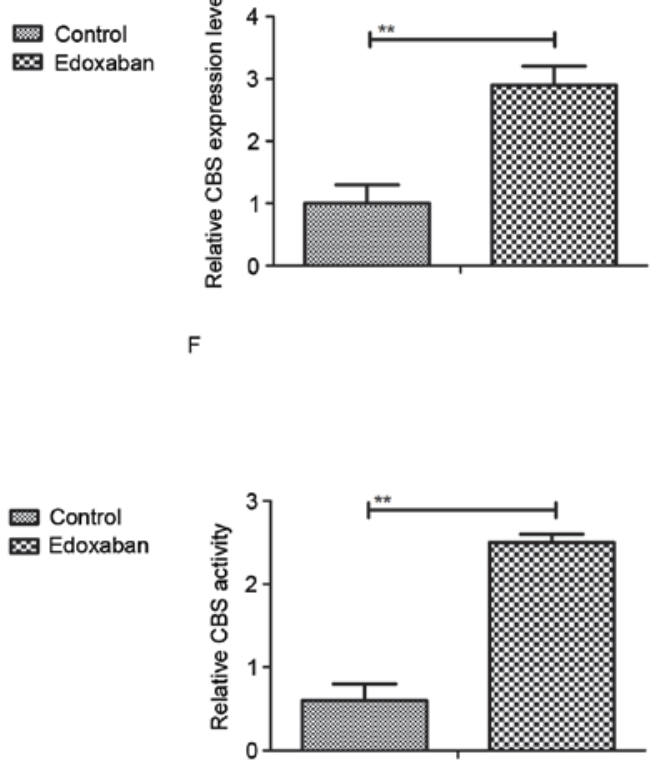

Control

Edoxaban

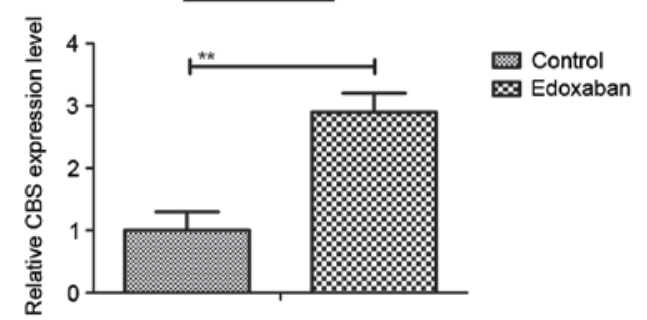

$\mathrm{F}$

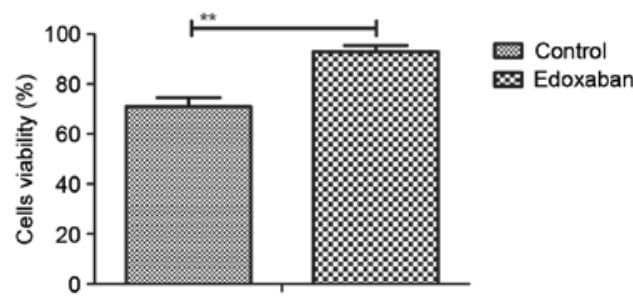

Figure 2. Edoxaban mediates expression levels of hydrogen sulfide in vein endothelial cells. Changes in (A) hydrogen sulfide and (B) homocysteine mRNA levels in vein endothelial cells following treatment with edoxaban. (C) ETS, (D) CBS and (E) CGL expression levels in vein endothelial cells following treatment with edoxaban. ( $F$ and G) CBS and CGL activities in vein endothelial cells after treatment with edoxaban. (H) Activity of vein endothelial cells following $48 \mathrm{~h}$ treatment of edoxaban. The data are presented as the mean \pm standard error of the mean of three independent experiments. ${ }^{* *} \mathrm{P}<0.01$ as indicated. ETS, E26 transformation-specific; CBS, cystathionine $\beta$-synthase; CGL, cystathionine $\gamma$-lyase.

surgery (32). These data suggested that edoxaban can improve ferric chloride-induced venous thrombosis via regulation of hydrogen sulfide and homocysteine activities through the MMP-9-induced PI3K/AKT signaling pathway.

A previous clinical trial demonstrated that hydrogen sulfide and homocysteine activities serve a crucial role in the progression of stroke and venous thrombosis (33). Reports have revealed that methionine metabolism disorder could lead to the upregulation of homocysteine in vein endothelial cells in patients with venous thrombosis $(34,35)$. The current investigations have exhibited that hydrogen sulfide and homocysteine activities and expression levels are downregulated in patients or animals with venous thrombosis. A previous study indicated that ADP can induce platelet aggregation and further leads to thrombin (36). Additional studies have explored the antithrombotic therapy targeting for ADP to improve 

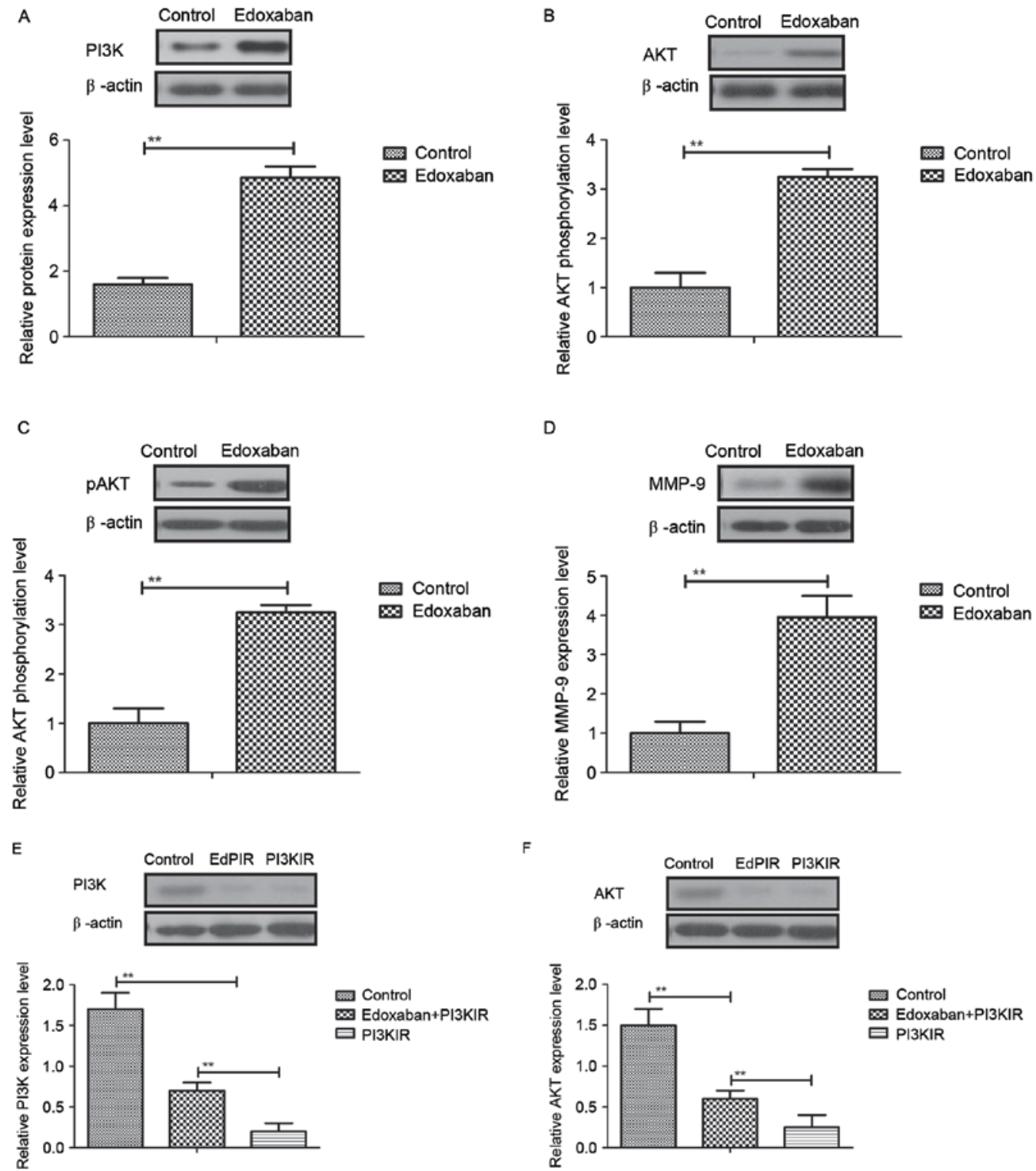

G
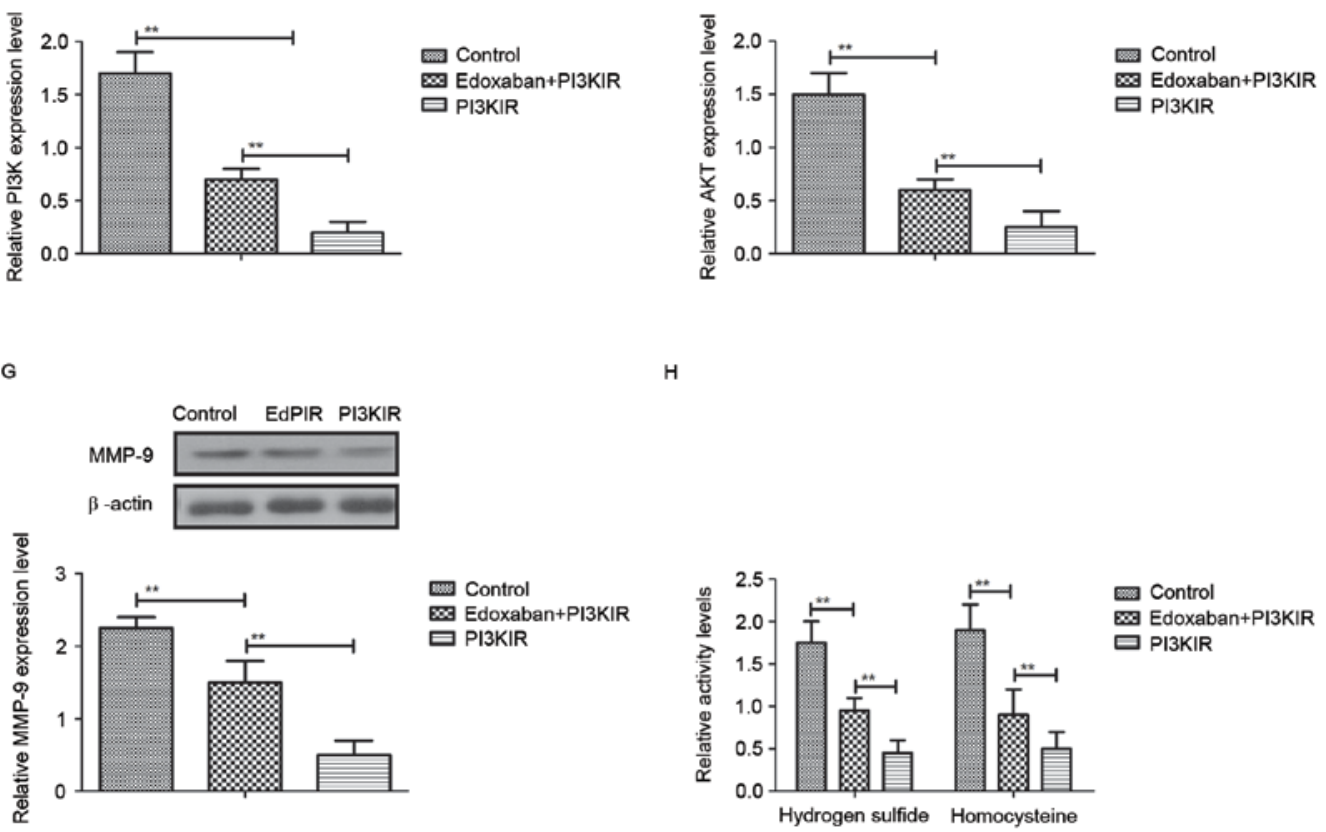

Figure 3. Edoxaban regulates activity of vein endothelial cells through the MMP9-induced PI3K/AKT signaling pathway. Expression levels of (A) PI3K and (B) AKT in edoxaban-treated microvascular endothelial cells. (C) Phosphorylation of AKT in edoxaban-treated microvascular endothelial cells. (D) MMP-9 expression levels in edoxaban-treated microvascular endothelial cells. (E) PI3K, (F) AKT and (G) MMP-9 expression levels in microvascular endothelial following treatment with PI3KIR. (H) Hydrogen sulfide and homocysteine activities in microvascular endothelial following treatment with PI3KIR. The data are presented as the mean \pm standard error of the mean of three independent experiments. ${ }^{* *} \mathrm{P}<0.01$ as indicated. PI3K, phosphoinositide 3-kinase; PI3KIR, PI3K inhibitor; AKT, protein kinase B; MMP-9, matrix metalloproteinase-9.

the ischemic disorders $(37,38)$. PAIs are another type of pro-thrombosis factor and Lenicek Krleza et al (39) indicated that upregulation of PAIs contribute to deep venous thrombosis with consequent pulmonary embolism in a case report. 
A

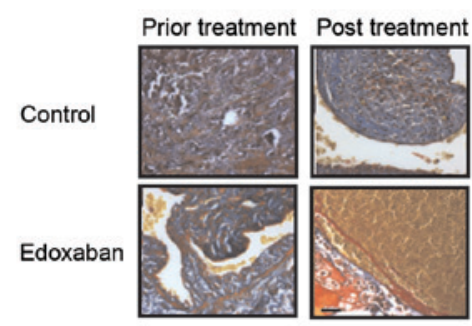

C

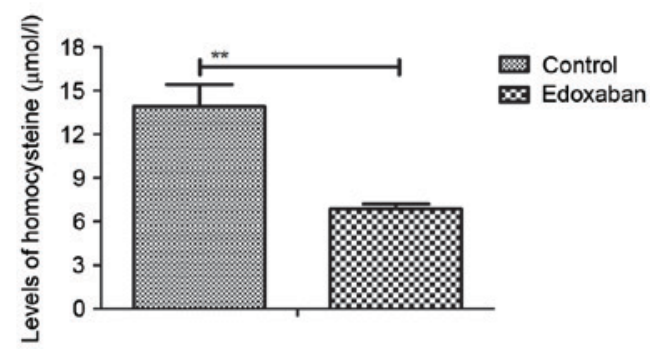

E
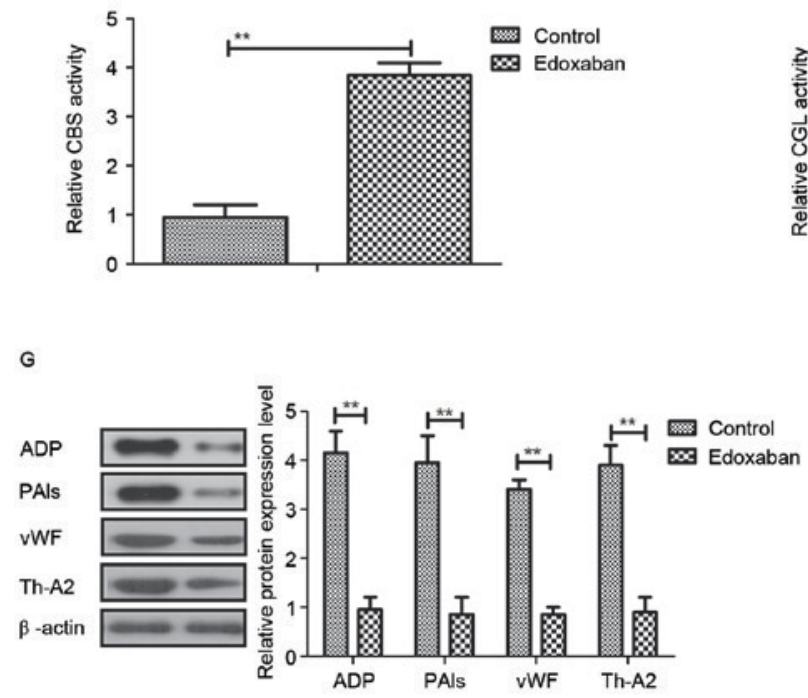

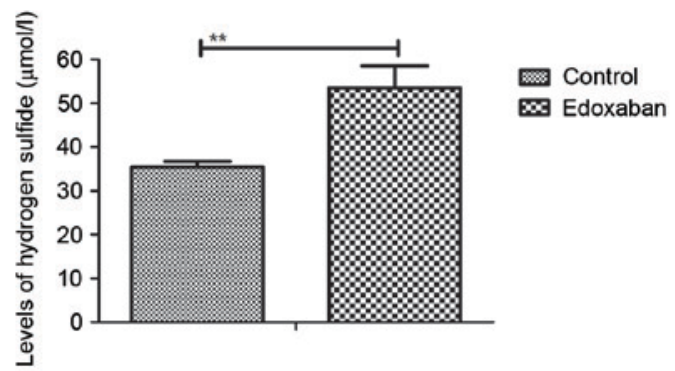

D
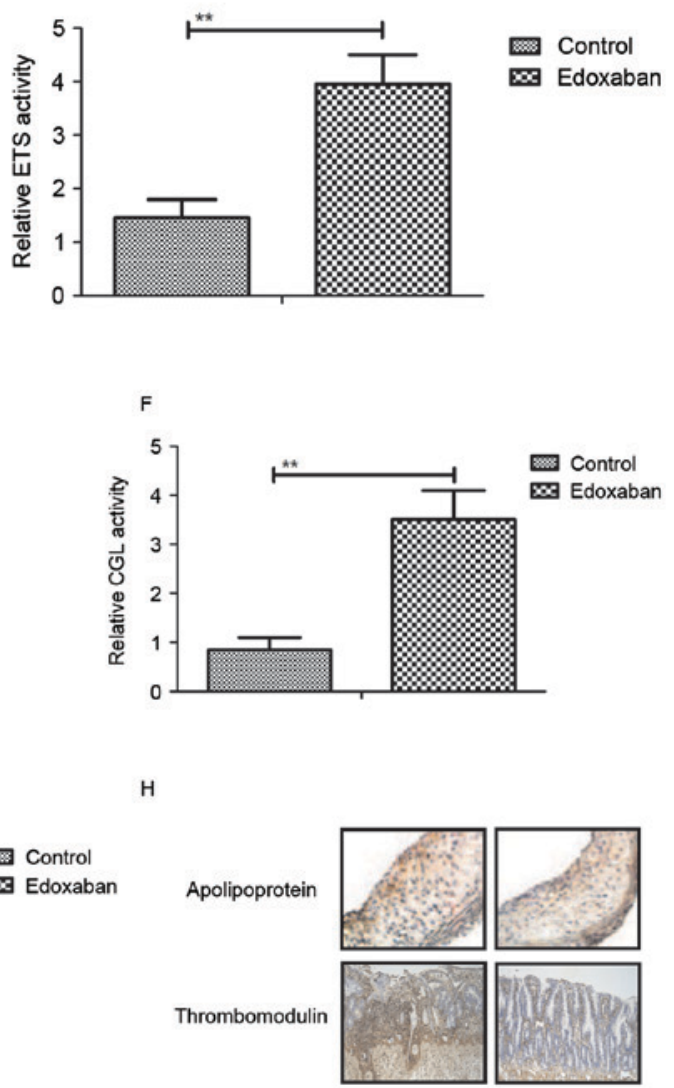

Figure 4. In vivo effects of edoxaban in rats with ferric chloride-induced venous thrombosis. (A) Venous lesions in rat with ferric chloride-induced venous thrombosis following receiving edoxaban treatment (magnification, $\mathrm{x} 40$ ). Plasma concentration levels of (B) hydrogen sulfide and (C) homocysteine in rats with ferric chloride-induced venous thrombosis treated by edoxaban. (D) ETS, (E) CBS and (F) CGL activities in microvascular endothelial cells in rats with ferric chloride-induced venous thrombosis treated by edoxaban. (G) ADP, PAIs, vWF and Th-A2 protein levels in microvascular endothelial cells following edoxaban treatment. $(\mathrm{H})$ Analysis of apolipoprotein and thrombomodulin expression levels in microvascular endothelial cells following treatment with edoxaban (magnification, $\mathrm{x} 40$ ). The data are presented as the mean \pm standard error of the mean of three independent experiments. ${ }^{* *} \mathrm{P}<0.01$ as indicated. ETS, E26 transformation-specific; PAIs, plasminogen activator inhibitors; vWF, von Willebrand factor; Th-A2, thromboxane-A2; CBS, cystathionine beta-synthase; CGL, cystathionine $\gamma$-lyase.

In addition, Yaroglu Kazanci et al (40) have indicated that PAI concentration levels were increased in patients with cerebral infarction and femoral venous thrombosis. Polymorphisms and mutations in the vWF gene and its plasma levels in patients with deep venous thrombosis have been investigated and presented an upregulation trend in patients with venous thrombosis (41). Additionally, TH-A2 protein levels also can affect the activation of platelet in venous thrombosis (42). The results have indicated that Edoxaban can improve expression levels of ADP, PAIs, vWF and TH-A2 protein levels both in vitro and in vivo. Notably, the data suggested that edoxaban regulates these thrombosis factors through the MMP9-inducedPI3K/AKT signaling pathway.

A previous report indicated that the PI3K/AKT signaling pathway serve multiple functions in the progression of disease occurrence (43). Edoxaban treatment increases PI3K and AKT expression levels in microvascular endothelial cells and rat with ferric chloride-induced venous thrombosis. 
Although the safety profile of edoxaban has been investigated in previous reports, the edoxaban-mediated PI3K/AKT signaling pathway has not been analyzed in previous studies. Guidetti et al (44) suggested that PI3K/AKT is stimulated by integrin engagement, further inhibiting activation of platelets in thrombus formation and stabilization, which highlights the possibility of venous thrombosis and anti-thrombotic therapeutic strategies. Su et al (45) also indicated that human cathelicidin LL-37 can inhibit the aggregation of platelets and further lead to inhibition of thrombosis via Src/PI3K/Akt signaling. Blood activity of platelets serves a crucial role in hemostasis and formation of thrombosis (46). In the present analysis, the authors reported that PI3K and AKT expression levels were upregulated by edoxaban through MMP-9 expression. However, inhibition of PI3K by its inhibitor suppresses PI3K and AKT expression levels and inhibits hydrogen sulfide and homocysteine activities in microvascular endothelial cells.

In conclusion, these novel findings revealed interesting insights to explore more efficient preclinical mechanism and therapeutic strategies of venous thrombosis. The results not only provide preclinical and experimental evidences to support the efficacy of edoxaban, but also elaborate the molecular mechanism of edoxaban-mediated changes of hydrogen sulfide and homocysteine through the PI3K/AKT signaling pathway in ferric chloride-induced venous thrombosis. The results indicated that edoxaban can improve the venous lesions by upregulating hydrogen sulfide and homocysteine activities via the MMP-9-mediated PI3K/AKT signaling pathway, suggesting edoxaban may be an efficient anti-thrombosis agent for the treatment of venous thrombosis in the clinic.

\section{Acknowledgements}

The present study was supported by the National Natural Science Foundation of China (grant no. U1503121).

\section{References}

1. Shlebak A: Antiphospholipid syndrome presenting as cerebral venous sinus thrombosis: A case series and a review. J Clin Pathol 69: 337-343, 2016.

2. Samos M, Bolek T, Ivanková J, Stančiaková L, Kovář F, Galajda P, Kubisz P, Staško J and Mokáň M: Heparin induced thrombocytopenia presenting with deep venous thrombosis and pulmonary embolism successfully treated with rivaroxaban: Clinical case report and review of current experiences J Cardiovasc Pharmacol 68: 391-394, 2016.

3. Jaqua NT, Stratton A, Yaccobe L, Tahir U, Kenny P and Kerns T: A review of the literature on three extraintestinal complications of ulcerative colitis: An ulcerative colitis flare complicated by Budd-Chiari syndrome, cerebral venous thrombosis and idiopathic thrombocytopenia. Acta Gastroenterol Belg 76: 311-316, 2013.

4. Barco S, Atema JJ, Coppens M, Serlie MJ and Middeldorp S: Anticoagulants for the prevention and treatment of catheter-related thrombosis in adults and children on parenteral nutrition: A systematic review and critical appraisal. Blood Transfus 15: 369-377, 2017.

5. Hawbaker S: Venous thromboembolism in the cancer population: Pathology, risk, and prevention. J Adv Pract Oncol 3: 23-33, 2012.

6. Vitale C, D'Amato M, Calabrò P, Stanziola AA, Mormile M and Molino A: Venous thromboembolism and lung cancer: A review. Multidiscip Respir Med 10: 28, 2015.

7. Ungprasert P, Tanratana P and Srivali N: Autoimmune hemolytic anemia and venous thromboembolism: A systematic review and meta-analysis. Thromb Res 136: 1013-1017, 2015.
8. Fernandez MM, Hogue S, Preblick R and Kwong WJ: Review of the cost of venous thromboembolism. Clinicoecon Outcomes Res 7: 451-462, 2015.

9. Corral J, Huntington JA, González-Conejero R, Mushunje A, Navarro M, Marco P, Vicente V and Carrell RW: Mutations in the shutter region of antithrombin result in formation of disulfide-linked dimers and severe venous thrombosis. J Thromb Haemost 2: 931-939, 2004.

10. Vaya A, Gómez I, Mira Y, Ferrando F and Corella D: Homocysteine levels in patients with deep vein thrombosis lacking thrombophilic defects. Thromb Haemost 99: 1132-1134, 2008.

11. Xue M, Yin H, Zhang L, Guo C, Jiang Y, Wu C, Li X and Chen K: Dynamic expression of the main related indicators of thrombosis, inflammatory reaction and tissue damage in a rat model of myocardial infarction. Mol Med Rep 4: 693-696, 2011.

12. Cao H, Zhang L, Sun ZB, Cheng XH, Zhang Y and Zou HB: Salvia miltiorrhiza prevents deep vein thrombosis via antioxidative effects in endothelial cells. Mol Med Rep 11: 3593-3600, 2015.

13. Li G, Han ZL, Dong HG, Zhang X, Kong XQ and Jin X: Platelet endothelial cell adhesion molecule-1 gene $125 \mathrm{C} / \mathrm{G}$ polymorphism is associated with deep vein thrombosis. Mol Med Rep 12: 2203-2210, 2015.

14. Ye S, Mao B, Yang L, Fu W and Hou J: Thrombosis recanalization by paeoniflorin through the upregulation of urokinase-type plasminogen activator via the MAPK signaling pathway. Mol Med Rep 13: 4593-4598, 2016.

15. Emerson M: Hydrogen sulfide and platelets: A possible role in thrombosis. Handb Exp Pharmacol 230: 153-162, 2015.

16. Ghaznavi H, Soheili Z, Samiei S and Soltanpour MS: Plasma homocysteine levels, methylene tetrahydrofolate reductase A1298C gene polymorphism and risk of retinal vein thrombosis. Blood Coagul Fibrinolysis 27: 679-683, 2016.

17. Qin YR, You SJ, Zhang Y, Li Q, Wang XH, Wang F, Hu LF and Liu CF: Hydrogen sulfide attenuates ferric chloride-induced arterial thrombosis in rats. Free Radic Res 50: 654-665, 2016.

18. Ekim M, Ekim H, Yilmaz YK, Kulah B, Polat MF and Gocmen AY: Study on relationships among deep vein thrombosis, homocysteine and related B group vitamins. Pak J Med Sci 31: 398-402, 2015.

19. Fay WP: Homocysteine and thrombosis: Guilt by association? Blood 119: 2977-2978, 2012.

20. Di Minno MN, Tremoli E, Coppola A, Lupoli R and Di Minno G: Homocysteine and arterial thrombosis: Challenge and opportunity. Thromb Haemost 103: 942-961, 2010.

21. Mahadeo KM, Dhall G, Panigrahy A, Lastra C and Ettinger LJ: Subacute methotrexate neurotoxicity and cerebral venous sinus thrombosis in a 12-year-old with acute lymphoblastic leukemia and methylenetetrahydrofolate reductase (MTHFR) C677T polymorphism: Homocysteine-mediated methotrexate neurotoxicity via direct endothelial injury. Pediatr Hematol Oncol 27: 46-52, 2010.

22. Ravari H, Zafarghandi MR, Alvandfar D and Saadat S: Serum homocysteine in deep venous thrombosis, peripheral atherosclerosis and healthy Iranians: A case-control study. Pak J Biol Sci 12: 1019-1024, 2009.

23. Loscalzo J: Homocysteine-mediated thrombosis and angiostasis in vascular pathobiology. J Clin Invest 119: 3203-3205, 2009.

24. Livak KJ and Schmittgen TD: Analysis of relative gene expression data using real time quantitative PCR and the 2(Delta Delta C(T)) method. Methods 25: 402-408, 2001.

25. Srivastava AK, Kalita J, Haris M, Gupta RK and Misra UK: Radiological and histological changes following cerebral venous sinus thrombosis in a rat model. Neurosci Res 65: 343-346, 2009.

26. Weeks CL, Singh S, Madzelan P, Banerjee R and Spiro TG: Heme regulation of human cystathionine beta-synthase activity: Insights from fluorescence and Raman spectroscopy. J Am Chem Soc 131: 12809-12816, 2009.

27. Cai J, Shi X, Wang H, Fan J, Feng Y, Lin X, Yang J, Cui Q, Tang C, Xu G and Geng B: Cystathionine $\gamma$ lyase-hydrogen sulfide increases peroxisome proliferator-activated receptor $\gamma$ activity by sulfhydration at C139 site thereby promoting glucose uptake and lipid storage in adipocytes. Biochim Biophys Acta 1861: 419-429, 2016.

28. Pan EY and Sobieraj DM: Considerations for prescribing target-specific oral anticoagulants in the setting of renal dysfunction or drug interactions. Conn Med 80: 105-111, 2016. 
29. Senger S, Keiner D, Hendrix P and Oertel J: New target-specific oral anticoagulants and intracranial bleeding: Management and outcome in a Single-Center case series. World Neurosurg 88: 132-139, 2016

30. Kundu A, Sen P, Sardar P, Chatterjee S, Kapoor A and McManus DD: Intracranial hemorrhage with target specific oral anticoagulants in patients with atrial fibrillation: An updated meta-analysis of randomized controlled trials. Int J Cardiol 203: 1000-1002, 2016.

31. Hurst KV, O'Callaghan JM and Handa A: Risk impact of edoxaban in the management of stroke and venous thromboembolism. Vasc Health Risk Manag 12: 329-335, 2016.

32. Partida RA and Giugliano RP: Edoxaban: Pharmacological principles, preclinical and early-phase clinical testing. Future Cardiol 7: 459-470, 2011.

33. Liu XQ, Liu XQ, Jiang P, Huang $H$ and Yan Y: Plasma levels of endogenous hydrogen sulfide and homocysteine in patients with Alzheimer's disease and vascular dementia and the significance thereof. Zhonghua Yi Xue Za Zhi 88: 2246-2249, 2008 (In Chinese).

34. Obeid OA, Johnston K and Emery PW: Plasma taurine and cysteine levels following an oral methionine load: Relationship with coronary heart disease. Eur J Clin Nutr 58: 105-109, 2004.

35. Klerk M, Lievers KJ, Kluijtmans LA, Blom HJ, den Heijer M, Schouten EG, Kok FJ and Verhoef P: The $2756 \mathrm{~A}>\mathrm{G}$ variant in the gene encoding methionine synthase: Its relation with plasma homocysteine levels and risk of coronary heart disease in a Dutch case-control study. Thromb Res 110: 87-91, 2003.

36. Tohti I, Tursun M, Umar A, Turdi S, Imin H and Moore N Aqueous extracts of Ocimum basilicum L. (sweet basil) decrease platelet aggregation induced by ADP and thrombin in vitro and rats Arterio-venous shunt thrombosis in vivo. Thromb Res 118: 733-739, 2006

37. Zhang L, Du JR, Wang J, Yu DK, Chen YS, He Y and Wang CY: Z-ligustilide extracted from Radix Angelica sinensis decreased platelet aggregation induced by ADP ex vivo and Arterio-venous shunt thrombosis in vivo in rats. Yakugaku Zasshi 129: 855-859, 2009.

38. Bernat A, Vallee E, Maffrand JP and Gordon JL: The role of platelets and ADP in experimental thrombosis induced by venous stasis in the rat. Thromb Res 52: 65-70, 1988.
39. Lenicek Krleza J, Jakovljevic G, Bronic A, Coen Herak D, Bonevski A, Stepan-Giljevic J and Roic G: Contraception-related deep venous thrombosis and pulmonary embolism in a 17-year-old girl heterozygous for factor $\mathrm{V}$ leiden, prothrombin G20210A mutation, MTHFR C677T and homozygous for PAI-1 mutation: Report of a family with multiple genetic risk factors and review of the literature. Pathophysiol Haemost Thromb 37: 24-29, 2010.

40. Yaroglu Kazanci S, Yesilbas O, Ersoy M, Kihtir HS, Yildirim HM and Sevketoglu E: Cerebral infarction and femoral venous thrombosis detected in a patient with diabetic ketoacidosis and heterozygous factor V Leiden G1691A and PAI-1 4G/5G mutations. J Pediatr Endocrinol Metab 28: 1183-1186, 2015.

41. Bittar LF, de Paula EV, Mello TB, Siqueira LH, Orsi FL and Annichino-Bizzacchi JM: Polymorphisms and mutations in vWF and ADAMTS13 genes and their correlation with plasma levels of FVIII and vWF in patients with deep venous thrombosis. Clin Appl Thromb Hemost 17: 514-518, 2011.

42. Tarantino E, Amadio P, Squellerio I, Porro B, Sandrini L, Turnu L, Cavalca V, Tremoli E and Barbieri SS: Role of thromboxane-dependent platelet activation in venous thrombosis: Aspirin effects in mouse model. Pharmacol Res 107: 415-425, 2016.

43. Huang XP, Ding H, Lu JD, Tang YH, Deng BX and Deng CQ Autophagy in cerebral ischemia and the effects of traditional Chinese medicine. J Integr Med 13: 289-296, 2015.

44. Guidetti GF, Canobbio I and Torti M: PI3K/Akt in platelet integrin signaling and implications in thrombosis. Adv Biol Regul 59: 36-52, 2015

45. Su W, Chen Y, Wang C, Ding X, Rwibasira G and Kong Y: Human cathelicidin LL-37 inhibits platelet aggregation and thrombosis via Src/PI3K/Akt signaling. Biochem Biophys Res Commun 473: 283-289, 2016.

46. McFadyen J and Peter K: Forget about thrombosis: Platelets and Alzheimer's disease, yet another sticky situation. Sci Signal 9: fs9, 2016. 